\title{
Study on follicular fluid metabolomics components at different ages based on lipid metabolism
}

\author{
Xingxing Zhang ${ }^{1 \dagger}$, Tianqi Wang ${ }^{2 \dagger}$, Jingyan Song ${ }^{3}$, Jifeng Deng ${ }^{4}$ and Zhengao Sun $^{5^{*}}$ (D)
}

\begin{abstract}
Background: Follicular fluid is an important external environment for the growth and development of oocytes. A thorough identification of specific components in follicular fluid can better the existing understand of intracellular signal transduction and reveal potential biomarkers of oocyte health in women undergoing assisted reproductive therapy. To study on follicular fluid metabolomics components at different ages based on lipid metabolism, we have adopted a new method of SWATH to MRM(the sequential window acquisition of all theoretical fragment-ion spectra to multiple reaction monitor)metabolomics to provide extensive coverage and excellent quantitative data. This was done to investigate the differences in follicular fluid of patients undergoing in vitro fertilization (IVF) and embryo transfer in different age groups and to further explore the relationship between follicular fluid, age and reproductive function.
\end{abstract}

Method: A combination of Ultra-high-performance liquid chromatography and high resolution mass spectrometry techniques were used to analyze the follicular fluid of 230 patients enrolled for the IVF cycle. The patients were of different ages grouped into two groups:the younger and older patients.The obtained multidimensional chromatographic data were processed by principal component analysis (PCA) and partial least squares discriminant analysis (PLS-DA). The charge ratios and mass numbers enabled for the identification of different fragments in the samples. Matching information obtained through database search and the fragment information obtained by fragment ion scan structurally identified substances in the samples. This was used to determine the differential compounds.

Results: The quality of oocytes decline with age,and the lipid composition in follicular fluid also changes, The lipid metabolism that changes with age may be related to the quality of oocytes.The main differences were in lipid metabolites. Some were up-regulated: Arachidonate, LysoPC(16:1), LysoPC(20:4) and LysoPC(20:3) while others were down-regulated: LysoPC(18:3) and LysoPC(18:1).

(Continued on next page)

\footnotetext{
*Correspondence: sunzhengao77@126.com

${ }^{+}$Xingxing Zhang and Tianqi Wang contributed equally to this work.

${ }^{5}$ Reproductive and Genetic Center of Integrated Traditional and Western

Medicine, The Affiliated Hospital of Shandong University of Traditional

Chinese Medicine, Jinan 250011, China

Full list of author information is available at the end of the article
}

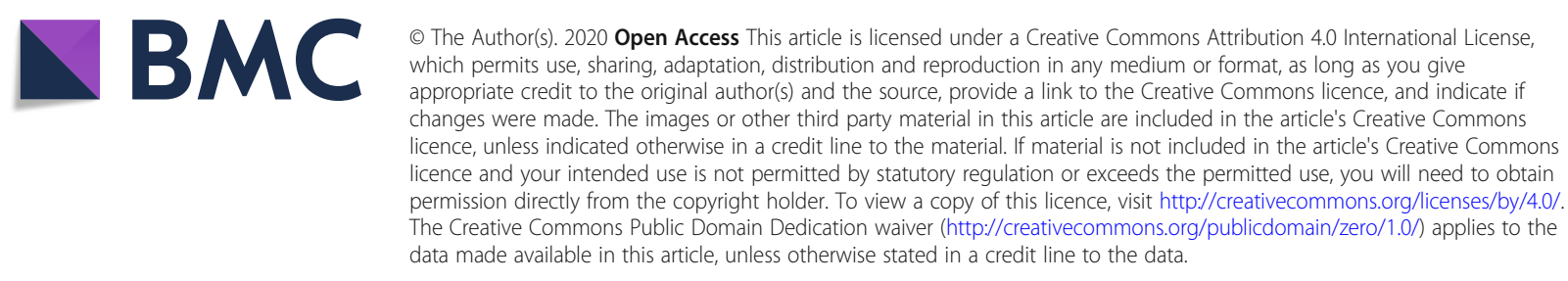


(Continued from previous page)

Conclusions: Metabolomic analysis of follicular fluid revealed that with the increase in age, several differential metabolites are at play. Among these metabolites, lipid metabolism undergoes significant changes that affect the development of oocytes thus causing reduced fertility in older women. These differential metabolites related to follicular development may provide possible detection and treatment targets for promoting oocyte health, and provide scientific basis for understanding the environment of oocyte development.

Keywords: Age, Oocytes, Lipid metabolism, Follicular fluid, Metabolomics

\section{Introduction}

Infertility is a worldwide problem affecting couples in their childbearing age. It is one of the three major diseases affecting human life and health. Development of social economy has led to a change in the concept of marriage and childbearing. The reproductive age of women has gone higher, and more older women are having pregnancy requirements. However, the reproductive capability of more older women is declining [1]. Finding solutions to fertility problems of aging women has become of paramount importance in reproductive medicine.However,the mechanism of age-related decline in reproductive function is still unclear. Numerous studies have shown that its mechanism may be related to a series of changes such as increased oocyte apoptosis, oxidative stress disorder, energy conversion disorder, and endocrine and metabolic abnormalities caused by age [2-6].

Follicular fluid is the culture medium in which an oocyte grows and differentiates. It directly or indirectly influences the fertility and up growth potency of oocytes thus indirectly affecting women's reproductive function.Changes in follicular microenvironment in older women may be responsible for altered protein function thus affecting the viability of oocytes. This undoubtedly causes adverse pregnancy outcomes $[7,8]$.

Metabolomics is an emerging omics technology developed after genomics and proteomics.It enables simultaneous qualitative and quantitative analysis of all metabolites of an organ, tissue or cells in a specific physiological period or condition in order to find target differential metabolites. It can be used for early diagnosis of disease, drug target discovery and disease mechanism research. Rapid development of metabolomics have enabled the use of follicular fluid as an objective evaluation index to reflect the quality of oocytes. This plays an important role in predicting the outcome of an in-vitro fertilization (IVF) cycle. Researchers in reproductive medicine have identified follicular fluid as a biomarker source for evaluating oocyte quality. Changes in its composition affect oocyte quality.Results obtained from the metabolomics analysis of follicular fluid have become an important indicator of clinical prediction of IVF outcomes [9].Previous studies have shown that the composition of the follicular fluid changes with age, which may be an important cause of the aging of oocytes [10]. These studies also revealed that the change process of female reproductive function may be the result of comprehensive regulation of multiple aspects and approaches. This study used metabolomics to analyze human follicular fluids (HFF) from patients of different ages.It opened up a new direction for exploring the mechanism of oocyte aging and provided new ideas and methods for treating senile infertility as well as improving IVF cycle outcomes.

\section{Materials and methods \\ Experimental chemicals}

The internal standards, including isotope-labeled Arachidonate (AA), isotope-labeled 16:1(d7) lysophosphatidylcholine(LPC),isotope-labeled 20:4(d7) LPC, isotopelabeled 20:3 (d7) LPC, isotope-labeled 18:3 (d7) LPC and Isotope-labeled 18:1 (d7) LPC, were purchased from Sigma-Aldrich (St. Louis, MO, USA). Distilled water was obtained from a Milli-Q system (Millipore, MA, USA). Chromatographic grade formic acid, acetonitrile and methanol were purchased from Fisher (Fairlawn, NJ, USA).

\section{Sample collection and preparation}

Source of cases: 230 cases of patients undergoing IVFET between August, 2016 and June, 2017, at the center of integrated traditional Chinese and western medicine reproductive and genetic center of affiliated hospital of Shandong university of traditional Chinese medicine. The study was approved by the Health Authorities and Ethics Committees of Shandong University of Traditional Chinese Medicine Affiliated Hospital. Diagnostic criteria: Previous pregnancy history. No contraception use for 12 months after pregnancy was regarded as secondary infertility [11].Inclusion criteria: Patients aged between 21 and 48 years meeting the diagnostic criteria of infertility caused by simple tubal factors. They also had to have no major gynecological diseases or other major diseases, have a body mass index (BMI) ranging between 18.5 and $24.9 \mathrm{~kg} / \mathrm{m}^{2}$ [12] and sign an informed consent.Exclusion criteria: Patients with infertility due to other factors such ascongenital ovarian dysplasia, serious 
malformation of reproductive organs, those with a major operation history, those with infertility due to male factors, those that have used hormone drugs within 3 months prior to the study. Grouping: Patients who met the inclusion criteria were first divided into two groups: a younger group (140 cases) of those aged between 28 and 34 years, and and an older group (90 cases) of those between 35 and 48 years. (World health care (WHO) and international association for obstetrics and gynecology scientific regulations). After identifying the major differences between the two groups, the 230 cases were reclassified into four groups: group A (54 cases) of those aged between 21 and 27 years old, group B (85 cases) of those aged between 28 and 34 years old, group C (51 cases) of those aged between 35 and 41 years old, and group D (40 cases) of those aged between 42 and 48 years old. This was done to observe the specific changes in follicular fluids between the four groups.

All subjects underwent controlled ovarian hyperstimulation according to our established protocols.All patients were enrolled in the controlled super-stimulatory antagonist program ( $\mathrm{GnRH}$-ant program).In the program, patients were first tested for serum sex hormone levels, ultrasonography was then done on the third day of their menstrual cycle to assess ovarian function. They then received recombinant follicle stimulating hormone (r-FSH, cognac) or/and injection with human gonadotropin (HMG) at astarting dose of $300 \mathrm{IU}$ per day for 4 days. After 4 days of follow-up, an ultrasound examination to monitor follicular development was done and adjustments of the $\mathrm{r}$-FSH, cognac/HMG dosage made. Once the dominant follicle had a diameter of 13 to $14 \mathrm{~mm}$, $0.25 \mathrm{mg}$ of leuprorelin Acetate per day was administered through injection. This was done until the day the injection with human chorionic gonadotropin (HCG) / recombinant-human chorionic gonadotropin ( $\mathrm{r}-\mathrm{HCG}$ ) was administered.

Follicular fluid collection: After the laboratory licked the eggs leave the first tube of FF place it in the original test tube let it stand for $10 \mathrm{~min}$ at room temperature take all the supernatant and place it in a $15 \mathrm{ml}$ centrifuge tube for centrifugation The constant rotation speed is $2000 \mathrm{r} / \mathrm{min}$ the centrifugation time is $5 \mathrm{~min}$ and the supernatant is taken up to $1.5 \mathrm{ml} \mathrm{EP}$ tube and stored in a $-80^{\circ} \mathrm{C}$ ultra-low temperature storage box for experiment and avoid repeated freezing or thawing.

\section{Serum hormone measurement and follicle calculation}

Circulating levels of hormones, including serum FSH, LH, E2, testosterone (detected at day 2), E2 and P (detected at hCG day), were measured using a radioimmunoassay method. The numbers of antral follicles were counted using ultrasonography on day 2 .

\section{Measurement of the reproducibility and accuracy of SWATH mass spectrometry}

Before analysis, eleven follicular fluid samples were thawed at room temperature for quality control (QC) (one QC after every four follicular fluid samples). First, samples were prepared by mixing $100 \mu \mathrm{L}$ of each individual follicular fluid samples. To reduce the effect of the solvent and obtain a good peak shape, a total volume of $150 \mu \mathrm{L}$ of follicular fluid or QC sample was mixed with $450 \mu \mathrm{L}$ of methanol $(\mathrm{v} / \mathrm{v}, 1: 3)$ containing isotopelabeled Arachidonate(AA),isotope-labeled 16:1(d7) lysophosphatidylcholine(LPC),isotope-labeled 20:4(d7) LPC, isotope-labeled 20:3 (d7) LPC, isotope-labeled 18:3 (d7) LPC and Isotope-labeled 18:1 (d7) LPC. Next, the mixture was vortexed for $10 \mathrm{~min}$ and centrifuged at $13000 \times \mathrm{g}$ for $20 \mathrm{~min}$ at $15^{\circ} \mathrm{C}$. The contents of the supernatant were analyzed using UPLC-Q-TOF.

\section{Method conditions}

Aliquots of $5 \mu \mathrm{L}$ of the supernatant were injected into the UPLC tandem Triple TOF 5600 system (SCIEX, CA, USA) in random order. A reverse-phase $2.1 * 100 \mathrm{~mm}$ ACQUITY $1.7 \mu \mathrm{m}$ C18 column (Waters, Ireland) was used for separation. A gradient mobile phase composed of $0.05 \%$ formic acid solution (A) and acetonitrile (B) was used and kept at $90 \% \mathrm{~A}$ for $0.5 \mathrm{~min}$, increased to 95\% B over the next $6.5 \mathrm{~min}$, and then returned to $90 \%$ A from $8.5 \mathrm{~min}$ to $8.6 \mathrm{~min}$. The total running time was $13 \mathrm{~min}$. The mass parameters were as follows: nebulizing gas, 55 psi; TIS gas, 55 psi; source temperature, $500^{\circ} \mathrm{C}$; and ion spray voltage, $5000 \mathrm{~V}$ with 35 psi curtain gas in positive mode and $-4000 \mathrm{~V}$ with 35 psi curtain gas in negative mode. The declustering potential and collision energy were set at $55 \mathrm{~V}$ and $40 \pm 20 \mathrm{~V}$, respectively, in positive mode $(-55 \mathrm{~V}$ and $-40 \pm 20 \mathrm{~V}$, respectively, in negative mode). The SWATH method with 20 variable isolation windows was performed in TOF MS full-scan mode and in TOF MS/MS product ion scan mode at $\mathrm{m} /$ z 50-1200 in Analyst TF 1.7.1 software.

\section{Data collection, processing and statistical analysis}

Analysis was done using SPSS 22.0 statistical software. $P$ value of less than $0.05(P<0.05)$ was considered statistically significant. The quantitative data of each group was normally distributed and hence the means and standard deviation was used for statistical description. The data was not statistically described using the median and quartile deviation. The changes in values before and after treatment in the two groups (the younger and the older) were compared with the normal distribution using the independent sample $t$ test, and the rank sum test without the normal distribution was compared. The count data of the two groups were statistically described 
using their frequency (composition ratio)and the chisquare test.

The multi-dimensional chromatographic data obtained were transformed into a matrix using metabolomic analysis methods: partial least squares-discriminant analysis (PCA and PLS-DA). In the scoring plot, each point represented a corresponding sample while in the loading plot, discrete points represented the variables separated in the score plot. Those with higher dispersion appeared more in the score plot. The $P$ value for each variable was determined using the $t$ test method. A variable with a $P$ value of more than 0.01 and less than $0.05,0.01<P<$ 0.05 was said to be significant while those with a $P$ value of less than $0.01, P<0.01$ was said to be very significant. According to the primary and secondary mass spectrum information of the groups' metabolic pathways, there were differences in charge ratio, mass number and isotope abundance.

\section{Results}

\section{Characteristics of patients}

From Table 1 , there were significant differences $(P<$ 0.01 ) in age, basic follicle stimulating hormone (bFSH), basic luteinizing hormone (bLH) and basic estradiol $\left(\mathrm{bE}_{2}\right)$ between the younger and the older group. The basic antral follicle count (bAFC) in the older group was significantly lower $(P<0.05)$ compared to that in the younger group This was an indication that the ovarian function of the older group was significantly low. However, there was no significant difference in Body Mass Index (BMI) between the two groups $(P>0.05)$.

\section{Ovulation outcomes}

Oocytes retrieval rate, 2PN cleavage rate and transferable embryo rate decreased with age. In all the difference between the younger and older group was statistically significant with $P$ values of $<0.001,0.002$ and $<0.001$ respectively. However, there was no significant difference $(P=0.077)$ in High-quality embryo rate between the younger age group and the older age group (Table 2).

Table 1 Between-group comparison of demographic and clinical characteristics

\begin{tabular}{llll}
\hline Item & Younger group & Older group & $P$-value \\
\hline Age (year) & $29.38 \pm 3.01$ & $40.51 \pm 3.47$ & $<0.001^{*}$ \\
BMI $\left(\mathrm{kg} / \mathrm{m}^{2}\right)$ & $21.62 \pm 6.67$ & $22.79 \pm 1.34$ & 0.05 \\
$\mathrm{bFSH}(\mathrm{IU} / \mathrm{L})$ & $7.49 \pm 1.88$ & $9.32 \pm 6.12$ & $<0.001^{*}$ \\
$\mathrm{bLH}(\mathrm{IU} / \mathrm{L})$ & $5.04 \pm 3.07$ & $5.70 \pm 6.70$ & $0.001^{*}$ \\
$\mathrm{bE}_{2}(\mathrm{pg} / \mathrm{ml})$ & $44.95 \pm 22.11$ & $50.66 \pm 36.30$ & $<0.001^{*}$ \\
bAFC $(\mathrm{n})$ & $15.65 \pm 5.83$ & $10.46 \pm 5.91$ & $0.001^{*}$ \\
\hline
\end{tabular}

*Significant at $P<0.05$
Significantly significant substance in follicular fluid between younger and older groups

It was found that there were Fourteen (14) different compounds in the two groups, 8 of which involved lipid metabolism as shown in Table 3.

To investigate the changes in lipid specific metabolites with age, we divided the patients into four groups based on age and analyzed their follicular fluid again. Six substances that were differentially regulated were detected: Arachidonate (AA), LysoPC(16:1), LysoPC(20:4), LysoPC(20:3), LysoPC(18:3) and LysoPC(18:1). The quantitative map results showed that there were four down-regulated substances:Arachidonate, LysoPC(16:1), LysoPC(20:4) and LysoPC(20:3) and two up-regulated substances: LysoPC(18:3) and LysoPC(18:1) (Table 4) (Fig. 1).

\section{Discussions}

In the present study, we differentiated the characteristics of metabolic ions in follicular fluid in the younger and older patients and identified several differential metabolites.

Metabonomic analysis of follicular fluid showed that several major metabolites changed with age, with some upregulated: arachidonic acid, LysoPC (16:1), LysoPC (20:4) and LysoPC (20:3), while others downregulated: LysoPC (18:3) and LysoPC (18:1). Among these metabolites, significant changes will take place in lipid metabolism, mainly characterized by the up-regulation of arachidonic acid, which affects oocyte development, while LPC participates in the regulation of follicular development and oocyte maturation, and its complex changes are closely related to follicular development. These differential metabolites related to follicular development may provide possible detection and therapeutic targets for promoting oocyte health. To provide scientific basis for understanding the environment of oocyte development.

Lipids is the general term used to refer to oils, fats, and lipids. They are important nutrients needed by the human body. They supply energy needed by the body as well as provide essential fatty acids for the body. Similarly, lipids are important energy support for oocyte growth and development; they participate in the construction of its membrane, regulate cell cycle and survival, malignant transformation and apoptosis [7-9].. It can be assumed that as age increases, lipid metabolism in the follicular fluid may cause a change in the oocyte. However,no study has shown an existing relationship between lipid metabolism oocyte aging. This study aimed to explore the role of lipid metabolism in oocyte aging in two different age groups through follicular fluid metabolomics It at the same time focused on opening up new directions for the treatment of senile infertility and 
Table 2 Between-group comparison of ovulation outcomes

\begin{tabular}{lllll}
\hline Item & Younger group & Older group & $x^{2}$ & $P_{\text {-value }}$ \\
\hline Oocytes retrieved rate (\%) & 88.0 & 66.5 & 162.8 & $<.001^{*}$ \\
2PN fertilization rate (\%) & 88.3 & 77.1 & 9.8 & $0.002^{*}$ \\
2PN cleavage rate (\%) & 98.0 & 84.3 & 51.3 & $<0.001^{*}$ \\
Transferable embryo rate (\%) & 72.8 & 55.7 & 39.1 & 3.25 \\
High-quality embryo rate (\%) & 45.0 & 38.2 & $0.001^{*}$ \\
\hline
\end{tabular}

*Significant at $P<0.05$

Note: Transferable embryo rate $=$ number of transferable embryos/2PN cleavage number; High-quality embryo rate $=$ number of high-quality embryos/2PN cleavage number

provide new ideas and methods to improve IVF outcome. This study suggests that a decline in female fertility due to age might be related to the change in lipid metabolism in the follicular fluid.

Arachidonate (AA) is an omega- 6 polyunsaturated fatty acid similar to the saturated peanut acid in peanut oil [1]. AA is an all-cis-5,8,11,14-eicosatetraenoic acid with a chemical formula: $\mathrm{CH} 3(\mathrm{CH} 2) 4(\mathrm{CH}=\mathrm{CH}$ $\mathrm{CH} 2) 4(\mathrm{CH} 2) 2 \mathrm{COOH}$. It is a saturated fatty acid containing four carbon-carbon double bonds and one carbon-oxygen double bond. It is widely distributed in the animal kingdom though it is found in small amounts in a variety of glycerides and glycerophospholipids. It has been found that AA and niclosamide potentially induce apoptosis through the common pathway of cytochrome $\mathrm{c}$ release from mitochondria.This is consistent with the view that niclosamide partially mediates cell death through arachidonic acid. Interestingly, AA has been found to increase mitochondrial permeability transition (PT) and cytochrome c release [13].

In addition to the environment in which lipid metabolism affect follicular development, lipid peroxidation is also closely related to follicular development [14]. One study found that the expressions of arachidonic acid 12-lipoxygenase (Alox12) and arachidonic acid 15lipoxygenase (Alox15) were significantly up-regulated in an obese diet reflecting an increase in lipid peroxidation. Lipid peroxidation is associated with many disease states associated with oxidative stress, aging and metabolic diseases [14]. Understanding of the multiple roles of lipoxygenases (other than in the classic arachidonic acid cascade) is increasing [15], creating a clearer picture of their role in generating the cellular redox balance.Lipoxygenases are prooxidative enzymes that by forming hydroperoxy lipids, can alter the redox state and gene expression pattern within the cell [16]. Apart from the implications for cellular redox balance, an increase in lipoxygenase activity also leads to greater oxidation of membrane lipids which in turn impairs the normal functions of the cell membrane and membrane-bound enzymes [17].Such impairments at the tissue level early in reproductive life may lead to increasing dysregulation of normal ovarian function later in life. Furthermore, there may be direct effects of Alox12 expression on both ovulation and follicular reserve. As an example,

Table 3 Significant differences in follicular fluid between younger and older groups

\begin{tabular}{lllll}
\hline Compound & Molecular Formula & Molecular weight & Charge-mass ratio & Pathway \\
\hline 4,5-Dihydroorotic acid & $\mathrm{C}_{5} \mathrm{H}_{6} \mathrm{~N}_{2} \mathrm{O}_{4}$ & 158.0327 & 159.0434 & Pyrimidine metabolism \\
Maleylacetoacetic acid & $\mathrm{C}_{8} \mathrm{H}_{8} \mathrm{O}_{6}$ & 200.0321 & 201.0591 & Aromatic nucleus metabolism \\
4-oxo-Retinoic acid & $\mathrm{C}_{20} \mathrm{H}_{26} \mathrm{O}_{3}$ & 314.1882 & 315.1931 & Vitamin metabolism \\
Arachidonate & $\mathrm{C}_{20} \mathrm{H}_{32} \mathrm{O}_{2}$ & 303.2321 & 304.2243 & Lipid metabolism \\
Nicotine glucuronide & $\mathrm{C}_{16} \mathrm{H}_{22} \mathrm{~N}_{2} \mathrm{O}_{6}$ & 338.1478 & 339.1428 & Glucose metabolism \\
DG(14:1(9Z)/22:2(13Z,16Z)/0:0) & $\mathrm{C}_{39} \mathrm{H}_{70} \mathrm{O}_{5}$ & 618.5223 & 619.5407 & Lipid metabolism \\
TG(18:1(11Z)/24:0/20:5(5Z,8Z,11Z,14Z,17Z)) & $\mathrm{C}_{65} \mathrm{H}_{114} \mathrm{O}_{6}$ & 990.8615 & Only in old woman & Lipid metabolism \\
Deoxycorticosterone & $\mathrm{C}_{21} \mathrm{H}_{30} \mathrm{O}_{3}$ & 330.2195 & 331.2139 & Biosynthesis of mineralocorticoid \\
LysoPC(14:0) & $\mathrm{C}_{22} \mathrm{H}_{46} \mathrm{NO}_{7} \mathrm{P}$ & 467.3012 & 468.3111 & Lipid metabolism \\
LysoPC(16:1) & $\mathrm{C}_{24} \mathrm{H}_{48} \mathrm{NO}_{7} \mathrm{P}$ & 495.3376 & 494.3222 & Lipid metabolism \\
LysoPC(18:0) & $\mathrm{C}_{26} \mathrm{H}_{54} \mathrm{NO}_{7} \mathrm{P}$ & 523.3638 & 524.3725 & Lipid metabolism \\
Phytosphingosine & $\mathrm{C}_{18} \mathrm{H}_{39} \mathrm{NO}_{3}$ & 317.293 & 318.3007 & Lipid metabolism \\
Phosphaticlylcholine(16:0/22:0) & $\mathrm{C}_{46} \mathrm{H}_{94} \mathrm{NO}_{7} \mathrm{P}$ & 323.1236 & Only in old woman & Lipid metabolism \\
5,6-Dihydrouridine & $\mathrm{C}_{9} \mathrm{H}_{14} \mathrm{~N}_{2} \mathrm{O}_{6}$ & 246.0852 & 247.074 & Nucleic acid metabolism \\
\hline
\end{tabular}


Table 4 the differential lipid metabolites in follicular fluid among the four groups

\begin{tabular}{|c|c|c|c|c|c|c|c|}
\hline Compound & $T_{R}(\min )$ & $\mathrm{m} / \mathrm{z}$ & Molecular Formula & Identity & MS/MS Fragments & $\begin{array}{l}\text { T-test } \\
\text { (p) }\end{array}$ & Pathway \\
\hline 1 & 8.9 & 303.2321 & $\mathrm{C}_{20} \mathrm{H}_{32} \mathrm{O}_{2}$ & Arachidonate $\downarrow$ & 59.0102; 205.1184; 259.1355; 285.0981 & $<0.01$ & Lipid metabolism \\
\hline 2 & 4.4 & 494.3222 & $\mathrm{C}_{24} \mathrm{H}_{48} \mathrm{NO}_{7} \mathrm{P}$ & LysoPC(16:1) $\downarrow$ & 104.1066; 184.0726; 311.2562; 476.3114 & $<0.01$ & Lipid metabolism \\
\hline 3 & 5.8 & 542.3185 & $\mathrm{C}_{28} \mathrm{H}_{50} \mathrm{NO}_{7} \mathrm{P}$ & LysoPC(20:4) $\downarrow$ & 86.0989; 146.9819; 337.2730; 483.2480 & $<0.01$ & Lipid metabolism \\
\hline 4 & 6.6 & 546.3489 & $\mathrm{C}_{28} \mathrm{H}_{52} \mathrm{NO}_{7} \mathrm{P}$ & LysoPC(20:3) $\downarrow$ & $104.1081 ; 184.0714 ; 341.3021 ; 487.2795$ & $<0.01$ & Lipid metabolism \\
\hline 5 & 4.7 & 518.3193 & $\mathrm{C}_{26} \mathrm{H}_{48} \mathrm{NO}_{7} \mathrm{P}$ & LysoPC(18:3) $\uparrow$ & 104.1072; 146.9842; 313.2700; 459.2479 & $<0.01$ & Lipid metabolism \\
\hline 6 & 5.3 & 522.3562 & $\mathrm{C}_{26} \mathrm{H}_{52} \mathrm{NO}_{7} \mathrm{P}$ & LysoPC(18:1) $\uparrow$ & $104.1058 ; 184.0724 ; 504.3413$ & $<0.01$ & Lipid metabolism \\
\hline
\end{tabular}

Note: “ $\downarrow$ ”: down-regulated with age; “^”: up-regulated with age

Alox12 is expressed in granulosa cells, thecal cells and follicular fluid at the time of ovulation in rats $[18,19]$, while inhibition of lipoxygenase can impair ovulation [19].Intriguingly,polymorphisms in the Alox12 gene have been linked with early age natural menopause in various human populations [20, 21], implying that their expression may be key to follicular reserve later in life.Estrogen biosynthesis and proteolysis are both important processes involved in ovarian follicular development though they may be influenced by cytochrome P450 (CYP)-dependent fatty acid metabolites. However, CYP-dependent lipid metabolism has not been characterized with respect to follicular maturation in vivo. The dynamic changes in follicular CYP-dependent arachidonic acid metabolites and their modulatory function in vascular models suggest roles for these metabolites in follicular maturation. This may include regulation of estradiol biosynthesis and preovulatory remodeling of the follicular wall that should be fully explored in future studies [22]. Folliculogenesis is an ordered sequence of oocyte development and maturation;it involves the proliferation and differentiation of granulosa cells and is crucial for mammalian reproduction [23]. During follicular development, a series of changes occur. First, the primordial follicle grows into a growth follicle with somatic cells proliferation after which the follicular antrum is formed as the ovarian granulosa cell undergoes proliferation and differentiation. The majority of follicles then become atresia and only few grow dramatically to become dominant follicles and ovulation follows. Follicular fluid (FF) within the follicular antrum is the microenvironment of oocyte. It contains hormones, growth factors, proteins and phospholipids which are partially produced by Granulosa cells(GCs). Phospholipids are the most abundant lipids in the cell membrane and they include Lysophosphatidic Acid (LPA), LysophosphatidylCholine (LPC), Sphingosine-1 Phosphates and Sphingophoryl Choline [24]. Some studies have shown that LPC is metabolized into LPA by phospholipase [25, 26].
A potential biological mechanism by which LPC could influences mitochondrial oxidative capacity is through its role in the synthesis pathway of cardiolipin. Cardiolipin is a unique dimeric phospholipid containing four fatty acid chains that are specific to mitochondria. Cardiolipin is an essential constituent of mitochondrial membranes [27]. LPC in human plasma can be generated by three activities: phospholipase A2 (PLA2) on phosphatidylcholine, by the activity of endothelial lipase (EL) including phospholipase A1 (PLA1) on high-density lipoprotein [28] and from phosphatidylcholine during the formation of cholesteryl esters. LPC is also found in membranes both mitochondria and endoplasmic reticulum [29, 30]. Synthesis of cardiolipin involves LPA and phosphatidic acid (PA). LPC can be hydrolyzed to LPA by autotaxin, a secreted glycoprotein that is widely expressed in tissues [31, 32]. LPA can also be formed from the acylation of glycerol-3-phosphate on the outer mitochondrial membrane. Two isoforms of acylglycerol3-phosphate acyltransferase (AGPAT); AGPAT 4 and 5 are located on the outer mitochondrial membrane and catalyze the acylation of LPA to PA [33]. PA is transferred to the inner mitochondrial membrane and is converted to nascent cardiolipin via CDP-glycerol, phosphatidylglycerophosphate, and phosphatidylglycerol. LPCs are highly mobile within intact cells and thus are good candidates for a cytoplasmic messenger that transduces signals to activate downstream processes and gene expression in the nucleus [34]. LPC can activate several second messengers; extracellular-signal-regulated kinases and protein kinase $\mathrm{C}$, which have been shown to be involved in the regulation of follicular development and oocyte maturation [35]. In humans, blood triglyceride levels tend to increase, while blood lysophosphatidylcholine levels tend to decrease with age. Specific sphingolipid and phospholipid blood profiles have also been shown to change with age and are associated with exceptional human longevity. These data suggest that lipidrelated interventions may improve human health span and that blood lipids likely represent a rich source of human aging biomarkers [36]. 


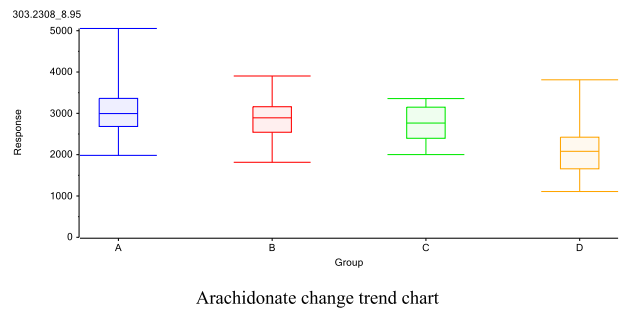

Note: "group A":aged between 21 and 27 years old;group B: aged between 28 and 34 years old;group C:aged between 35 and 41 years old;group D:aged between 42 and 48 years old.
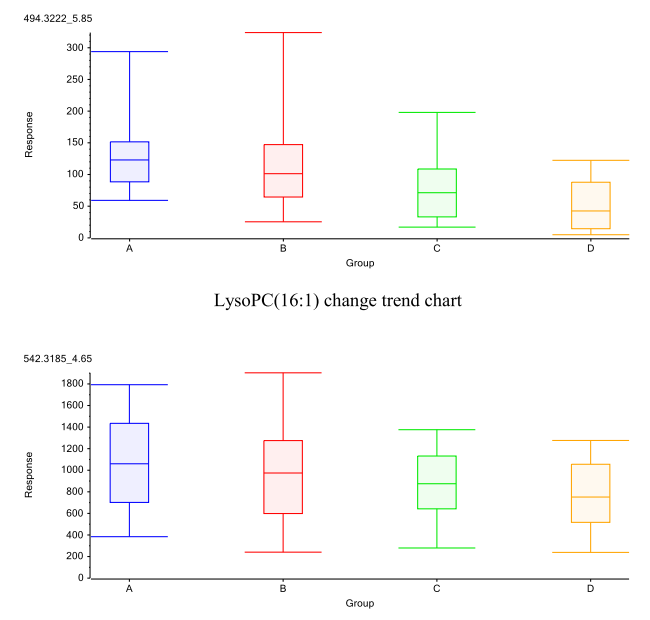

LysoPC(20:4) change trend chart

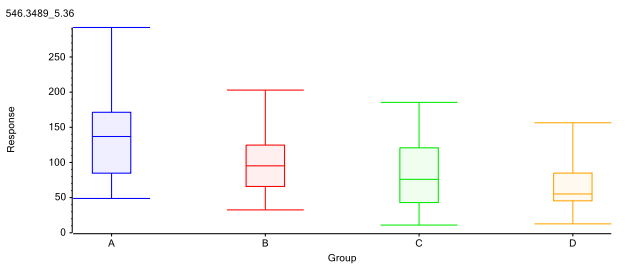

LysoPC(20:3) change trend chart

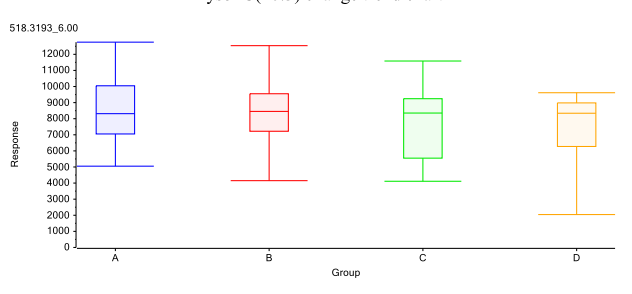

LysoPC(18:3) change trend chart

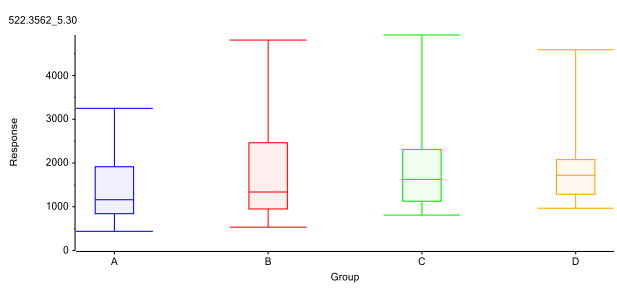

LysoPC(18:1) change trend chart
Fig. 1 Arachidonate change trend chart. Note: "group A":aged between 21 and 27 years old;group B: aged between 28 and 34 years old;group C:aged between 35 and 41 years old;group D:aged between 42 and 48 years old. LysoPC(16:1) change trend chart. LysoPC(20:4) change trend chart. LysoPC(20:3) change trend chart. LysoPC(18:3) change trend chart. LysoPC(18:1) change trend chart

\section{Conclusions}

Using advanced SWATH ${ }^{\mathrm{TM}}$-based mass spectrometry, we investigated metabolic changes in the follicular fluid of the younger and older patients.Metabonomic analysis of follicular fluid showed that several major metabolites changed with age, with some upregulated: arachidonic acid, LysoPC (16:1), LysoPC (20:4) and LysoPC (20:3), while others downregulated: LysoPC (18:3) and LysoPC (18:1). Among these metabolites, significant changes will take place in lipid metabolism, mainly characterized by the up-regulation of arachidonic acid, which affects oocyte development, while LPC participates in the regulation of follicular development and oocyte maturation, and its complex changes are closely related to follicular development. These differential metabolites related to follicular development may provide possible detection and therapeutic targets for promoting oocyte health. To provide scientific basis for understanding the environment of oocyte development.

\section{Abbreviations}

SWATH: Sequential window acquisition of all theoretical fragment-ion spectra; MRM: Multiple reaction monitor; PCA: Principal component analysis; PLSDA: Partial least squares discriminant analysis; FF: Human follicular fluids; AA: Arachidonate; LPC: Lysophosphatidylcholine; QC: Quality control; UPLCQ-TOF: Ultra-performance liquid chromatography quadrupole time-of-flight mass spectrometry; CYP: Cytochrome P450; GCs: Granulosa cells;

LPA: Lysophosphatidic Acid; PLA2: Phospholipase A2; EL: Endothelial lipase; PLA1: Phospholipase A1; PA: Phosphatidic acid; AGPAT: Acylglycerol-3phosphate acyltransferase

\section{Acknowledgements}

We are thankful to all the participants of the study.

\section{Authors' contributions}

XZ and TW performed the literature search, data analysis and interpretation, and wrote the manuscript. JS constructed the tables and Figures. JD performed the sample collection and data analysis. ZScontributed to the design of the research study and provided critical review of the manuscript. All authors read and approved the final manuscript.

\section{Funding}

This work was supported by the National Natural Science Fund Project (No. 81373676; No.81674018).

\section{Availability of data and materials}

The datasets used and analyzed during the current study are available from the corresponding author on reasonable request.

\section{Ethics approval and consent to participate}

This study was approved by the Health Authorities and Ethics Committees of Shandong University of Traditional Chinese Medicine Affiliated Hospital. All participants signed an informed consent form prior to the study.

Consent for publication

Not applicable. 


\section{Competing interests}

The authors declare that they have no competing interests.

\section{Author details}

'Maternity and Child Health Care of ZaoZhuang, ZaoZhuang 277100, Shandong, China. ${ }^{2}$ Traditional Chinese Medicine History and Literature, Institute for Literature and Culture of Chinese Medicine, Shandong University of Traditional Chinese Medicine, Jinan 250355, China. ${ }^{3}$ Department of Gynecology and Obstetrics of Traditional Chinese Medicine, The First Clinical College, Shandong University of Traditional Chinese Medicine, Jinan 250014, China. ${ }^{4}$ School of Bioscience \& Bioengineering, South China University of Technology, Guangzhou 510640, China. ${ }^{5}$ Reproductive and Genetic Center of Integrated Traditional and Western Medicine, The Affiliated Hospital of Shandong University of Traditional Chinese Medicine, Jinan 250011, China.

Received: 23 February 2020 Accepted: 24 April 2020

Published online: 12 May 2020

\section{References}

1. Vayena E, Rowe PJ, Griffin PDJH. Current practices and controversies in assisted reproduction; 2002.

2. Bruin JP, De DM, Spek ER, Posthuma G, Haaften M, Van LCWN, et al. Agerelated changes in the ultrastructure of the resting follicle pool in human ovaries. Biol Reprod. 2004;70(2):419-24.

3. Ling G, Honglin L, Xi G, Christina B, Moley KH, Qiang WJC, et al. Metabolic control of oocyte development: linking maternal nutrition and reproductive outcomes. Cell Mol Life Sci. 2015;72(2):251-71.

4. Teresa Almeida S, Shahy ES, St. John JC. Mitochondrial content reflects oocyte variability and fertilization outcome. Fertil Steril. 2006;85(3):584-91.

5. Jana SK, Narendra Babu K, Ratna C, Baidyanath C, Koel CJRT. Upper control limit of reactive oxygen species in follicular fluid beyond which viable embryo formation is not favorable. Reprod Toxicol. 2010;29(4):447-51.

6. Zhang HH, Ping YX, Wu J, Wei WZ, Xia MX, Xia YC, et al. Dehydroepiandrosterone improves follicular fluid bone morphogenetic protein-15 and accumulated embryo score of infertility patients with diminished ovarian reserve undergoing in vitro fertilization: a randomized controlled trial. J Ovarian Res. 2014;7(1):93.

7. Leanne PI, Zander-Fox DL, Lane M. Mitochondrial SIRT5 is present in follicular cells and is altered by reduced ovarian reserve and advanced maternal age. Reprod Fertil Dev. 2014;26(8):1072-83.

8. Jmc DLB, Boueilh T, Simard G, Boucret L, Ferré-L'Hotellier $V$, Tessier L, et al. Targeted metabolomics reveals reduced levels of polyunsaturated choline plasmalogens and a smaller dimethylarginine/arginine ratio in the follicular fluid of patients with a diminished ovarian reserve. Hum Reprod. 2017 32(11):2269-78.

9. Alberto R, Luisa DP, Simona C, Emanuela M, Marco M, Paolo R. Follicular fluid content and oocyte quality: from single biochemical markers to metabolomics. Reprod Biol Endocrinol. 2009:7(1):40.

10. Hułasstasiak M, Gawron A. Follicular atresia in the prepubertal spiny mouse (Acomys cahirinus) ovary. Apoptosis. 2011;16(10):967-75.

11. Zegers-Hochschild F, Adamson GD, Jd M, Ishihara O, Mansour R, Nygren K, et al. International Committee for Monitoring Assisted Reproductive Technology (ICMART) and the World Health Organization (WHO) revised glossary of ART terminology. Fertil Steril. 2009;92(5):0-1524.

12. Obesity: preventing and managing the global epidemic. Report of a WHO consultation. J World Health Organ Tech Rep Ser. 2000;894(i-xii):1-253.

13. Kumar R, Coronel L, Somalanka B, Raju A, Aning OA, An O, et al. Mitochondrial uncoupling reveals a novel therapeutic opportunity for p53-defective cancers[J]. Nat Commun. 2018;9(1):3931.

14. Aiken CE, Tarryadkins JL, Penfold NC, Dearden L, Ozanne SE. Decreased ovarian reserve, dysregulation of mitochondrial biogenesis, and increased lipid peroxidation in female mouse offspring exposed to an obesogenic maternal diet. FASEB J. 2016:30(4):1548-56.

15. Kuhn H, Banthiya S, Leyen KV. Mammalian lipoxygenases and their biological relevance $i$ s. Biochimica et Biophysica Acta (BBA)-Molecular and Cell Biology of Lipids. 2015;1851(4):308-30.

16. Forman HJ, Ursini F, Maiorino M. An overview of mechanisms of redox signaling. J Mol Cell Cardiol. 2014;73:2-9.

17. Buelna-Chontal M, Zazueta C. Redox activation of Nrf2 \& NF-kB: A double end sword? Cell Signal. 2013;25(12):2548-57.
18. Tanaka N, Espey LL, Okamura H. Increase in ovarian 15 hydroxyeicosatetraenoic acid during ovulation in the gonadotropin-primed immature rat. Endocrinology. 1989;125(3):1373-7..

19. Shiro K, Masako J, Hirosato E, Tomohiro Y, Mitsumori KJR. Inhibition of ovulation by a lipoxygenase inhibitor involves reduced cyclooxygenase-2 expression and prostaglandin E2 production in gonadotropin-primed immature rats. Reproduction. 2009;137(1):59-66.

20. Liu P, Lu Y, RJM-tJotNAMS. Alox12 gene is associated with the onset of natural menopause in white women. Menopause. 2010:17(1):152-6.

21. Geoffrey G, Jacques PJA, Signaling R. Redox regulation of nuclear posttranslational modifications during NF-kappaB activation. Antioxid Redox Signal. 2009;11(9):2209-22.

22. Newman JW, Stok JE, Vidal JD, Corbin CJ, Huang Q, Hammock BD, et al. Cytochrome p450-dependent lipid metabolism in preovulatory follicles[J]. Endocrinology. 2004;145(11):5097-105.

23. Zhang T, Shen W, De FM, Zhang XFJE, Mutagenesis M. Di(2ethylhexyl)phthalate: Adverse effects on folliculogenesis that cannot be negelected. Environ Mol Mutagen. 2016;57(8):579-88.

24. Ye X. Lysophospholipid signaling in the function and pathology of the reproductive system. Hum Reprod Update. 2008;14(5):519-36.

25. Ye X, Chun J. Lysophosphatidic acid (LPA) signaling in vertebrate reproduction. Trends Endocrinol Metab. 2010;21(1):17-24.

26. Sindhu R, Manoj G, Vishnu S, et al. Autotaxin-lysophosphatidic acid signaling in alzheimer's disease. Int J Mol Sci. 2018;19(7):1827.

27. Schlame M, Greenberg ML. Biosynthesis, remodeling and turnover of mitochondrial cardiolipin. Biochim Biophys Acta Mol Cell Biol Lipids. 2016; 1862(1):S138819811630230X

28. Semba RD, Shardell M, Trehan I, Moaddel R, Maleta KM, Ordiz Ml, et al. Metabolic alterations in children with environmental enteric dysfunction. Sci Rep. 2016;6(1):28009.

29. Tsalouhidou S, Argyrou C, Theofilidis G, Karaoglanidis D, Orfanidou E, Nikolaidis MG, et al. Mitochondrial phospholipids of rat skeletal muscle are less polyunsaturated than whole tissue phospholipids: implications for protection against oxidative stress. J Animal Sci. 2006;84(10):2818-25.

30. Veyrat-Durebex C, Bocca C, Chupin S, Kouassi NJ, Simard G, Lenaers G, et al. Metabolomics and lipidomics profiling of a combined mitochondrial plus endoplasmic reticulum fraction of human fibroblasts: a robust tool for clinical studies. J Proteome Res. 2017;17(1):745-50.

31. Rossman IJBMU. Normal Human Aging: The Baltimore Longitudinal Study of Aging[J]. 1986;25(6):661.

32. Trabado S, Alsalameh A, Croixmarie V, Masson P, Corruble E, Fève B, et al. The human plasma-metabolome: reference values in 800 French healthy volunteers; impact of cholesterol, gender and age. PloS One. 2017;12(3): e0173615

33. Gonzalez-Baro MR, Coleman RA. Mitochondrial acyltransferases and glycerophospholipid metabolism. BBA-Mol Cell Biol Lipids. 2016;1862(1): S1388198116301755.

34. Drissner D, Kunze G, Callewaert N, Gehrig P, Tamasloukht M, Boller T, et al. Lyso-phosphatidylcholine is a signal in the arbuscular mycorrhizal symbiosis. Science. 2007:318(5848):265-8.

35. Wen X, Kuang Y, Zhou L, Yu B, Chen Q, Fu Y, et al. Lipidomic components alterations of human follicular fluid reveal the relevance of improving clinical outcomes in women using progestin-primed ovarian stimulation compared to short-term protocol [J]. Med Sci Monit. 2018;24:3357-65.

36. Johnson AA, Stolzing A. The role of lipid metabolism in aging, lifespan regulation, and age-related disease. Aging Cell. 2019;18(6):e13048.

\section{Publisher's Note}

Springer Nature remains neutral with regard to jurisdictional claims in published maps and institutional affiliations. 